\title{
A posição do Brasil na corrida pelo etanol celulósico: mensuração por indicadores C\&T e programas de P\&D*
}

\section{Iraci Souza João}

Faculdade de Economia, Administração e Contabilidade de Ribeirão Preto (FEA-RP/USP)

Geciane Silveira Porto

Faculdade de Economia, Administração e Contabilidade de Ribeirão Preto (FEA-RP/USP)

\section{Simone Vasconcelos Ribeiro Galina}

Faculdade de Economia, Administração e Contabilidade de Ribeirão Preto (FEA-RP/USP)

\section{RESUMO}

Embora o Brasil domine a tecnologia de produção de etanol de primeira geração, esse está ameaçado devido à possibilidade de se produzir etanol celulósico em escala comercial competitivamente. Assim, os objetivos da pesquisa foram verificar os resultados de esforços científicos e tecnológicos dos países no desenvolvimento do etanol celulósico, enfocando redes de inovação, e comparar os dois principais programas brasileiros de $\mathrm{P} \& \mathrm{D}$ (Bioen e CTBE) com o dos Estados Unidos (Biomass Program), país referência. Para tal, foram levantados indicadores de C\&T nas bases Web of Science e Esp@cenet. Encontraram-se 78 solicitações de patentes e 320 artigos publicados. Nota-se considerável atraso brasileiro, com atuação regional do Estado e baixo envolvimento da iniciativa privada nas redes estudadas (duas empresas no

* As autoras agradecem os comentários e sugestões dos pareceristas anônimos da revista. Possíveis erros e incorreções desta versão final são de responsabilidade exclusiva das autoras. 
Brasil contra 117 nos Estados Unidos), o que reduz as chances do Brasil em dominar essa nova rota tecnológica.

Palavras-Chave | Etanol Celulósico; Redes de Inovação, Bioen-Fapesp; Biomass Program; CTBE.

Código JEL | 032.

\title{
The position of Brazil on the run for Cellulosic Ethanol: mensuration by S\&T and P\&D programs
}

\begin{abstract}
Although Brazil masters the technology of producing cutting edge ethanol, its position is jeopardized by the possibility of the production of cellulosic ethanol in commercial scale competitively. Therefore, the present research is intended for (i) verifying the results of scientific and technological efforts made by countries which develop cellulosic ethanol, (ii) focusing innovation networks, comparing Bioen and CTBE, the two main Brazilian P\&D programs to Biomass Program, to the United States, the reference country. It was listed science and technology indexes on Web of Science and Esp@cenet bases, and out of a total of 78 patent applications and 320 papers published. It is noticed a considerable backwardness in what concerns Brazil, with regional state operations and low involvement by the private sector on the networks studied, that is, two Brazilian companies and 117 American ones, which reduces the chances of Brazil to master this new technology path.
\end{abstract}

KeYwords | Cellulosic Ethanol; Innovation Networks; Bioen-Fapesp; Biomass Program; CTBE.

JEL-Code | 032. 


\section{Introdução}

As projeçōes para o fim das reservas naturais da principal fonte energética utilizada atualmente, o petróleo, e o consequente incremento da demanda por recursos energéticos sustentáveis têm colocado em voga a real necessidade da identificação e desenvolvimento de fontes alternativas de energias denominadas limpas e renováveis. Nesse contexto, o Brasil ocupa posição de destaque devido a fatores como o fato de sua matriz elétrica ser composta por aproximadamente $90 \%$ de hidroeletricidade (BRASIL, 2009) e o domínio da tecnologia de produção de etanol a partir da extração do caldo da cana-de-açúcar, por meio da fermentação alcoólica da sacarose, denominado de etanol de primeira geração.

Esse combustível, com potencial energético consideravelmente superior ao do milho, ${ }^{1}$ matéria-prima também utilizada para a produção de etanol, ganhou destaque no Brasil na década de 1970, diante da crise energética mundial que levou o país a criar o Programa Nacional do Álcool - Proálcool, a fim de reduzir a dependência brasileira em relação ao petróleo. A ação do programa fez do Brasil o segundo maior produtor mundial de etanol. Em 2009, aproximadamente 92\% dos veículos leves vendidos no país ${ }^{2}$ eram movidos a etanol ou então possuíam a tecnologia flexfuel (ANFAVEA, 2010).

Contudo, o domínio tecnológico e produtivo brasileiro pode ser afetado pela possibilidade de se produzir etanol a partir dos polissacarídeos da parede celular vegetal, chamado etanol celulósico, etanol lignocelulose, ou, ainda, de segunda geração, uma vez que o Brasil não possui o domínio dessa rota tecnológica. Esse tipo de etanol pode ser produzido a partir de seis diferentes grupos principais de matéria-prima: resíduos de culturas (bagaço da cana-de-açúcar, palha de milho, palha de trigo, palha de arroz, casca de arroz, etc.), madeira, resinosas (pinheiros), resíduos de celulose (jornal, papel de escritório e reciclado), herbáceos (feno de alfafa, gramíneas, cana, etc.) e resíduos urbanos (CARDONA; QUINTERO; PAZ, 2010).

Segundo Carbona, Quintero e Paz (2010), essas matérias-primas possuem até 75\% de celulose e hemicelulose que podem ser extraídas, fermentadas e transformadas em etanol. Contudo, os procedimentos conhecidos apresentam alto custo, o que inviabiliza a produção do etanol de segunda geração em escala comercial.

\footnotetext{
1 Para cada unidade de energia fóssil, pode-se produzir de 9 a 10 unidades de energia de etanol de cana-de-açúcar (MACEDO; SEABRA; SILVA, 2008) ou 1,3 unidade de energia de etanol de milho.

2 Exceto os movidos a diesel.
} 
Dessa forma, tal desafio tem mobilizado grande número de pesquisadores, empresas e agências de fomento em vários países do mundo, uma vez que, segundo a visão schumpeteriana, na maioria dos casos, quem introduz a tecnologia no mercado obtém maiores lucros, até a inovação se difundir entre os concorrentes ou a patente expirar (SCHUMPETER, 1984).

A organização desses diferentes agentes em redes surge como uma possibilidade de viabilizarem, cooperativamente, o processo de desenvolvimento da tecnologia necessária para inovação, uma vez que as dificuldades para obtenção de rota tecnológica do etanol celulósico são várias. Entre estas estão a conversão da energia armazenada na celulose em etanol, a concorrência com outras rotas tecnológicas para a captação de investimentos em P\&D e demanda de mercado, a necessidade de novas políticas públicas, o desenvolvimento de motores que permitam a máxima utilização desse combustível, etc.

A partir desse panorama, vale considerar que a estruturação em redes é indicada para situações em que se fazem necessárias a criação e a integração de diferentes habilidades e conhecimentos, a fim de se desenvolverem tecnologias complexas e introduzi-las no mercado mais rapidamente (GUARAU, 2005; RYCROFT; KASH, 2004), como no caso do etanol celulósico, em que a quebra da parede celular a baixo custo ainda demanda alto esforço em P\&D.

Para o Brasil, o desenvolvimento dessa tecnologia tem importância singular, pois representa a manutenção do domínio tecnológico e, consequentemente, dos benefícios advindos com a prática de inovação, ou seja, a continuidade da liderança produtiva e tecnológica em um setor estratégico para o país, que representou, em 2008, aproximadamente 1,5\% do PIB nacional (NEVES; TROMBIN; CONSOLI, 2009), a possibilidade de aprimorar a produção de etanol a partir da cana-de-açúcar, principal matéria-prima utilizada no país, e o desenvolvimento de novas fontes renováveis de energia.

Duas técnicas em especial têm recebido maior direcionamento dos investimentos de P\&D no Brasil e no mundo: a hidrólise ácida e a hidrólise enzimática, nas quais são utilizados, como agentes hidrolisantes, solventes ácidos e conjunto de enzimas, respectivamente, ocasionando o descolamento da lignina da celulose que é transformada em glicose, que, por sua vez, é fermentada, obtendo-se o álcool.

Diante desse cenário, é relevante entender quais são os principais países que vêm liderando a busca por uma rota tecnológica que viabilize a produção do etanol de segunda geração em escala comercial e avaliar os mecanismos adotados por eles em comparação com os do Brasil. Assim, este estudo se propõe a: 
- verificar os esforços científicos e tecnológicos dos países para o desenvolvimento do etanol celulósico, evidenciando onde eles são mais intensos;

- comparar os dois principais programas brasileiros de P\&D com aquele desenvolvido pelo país com participação mais destacada em termos científicos e tecnológicos (identificados na etapa A), sob o enfoque de redes de inovação.

O intuito é avaliar comparativamente o Brasil em relação aos principais países concorrentes pela busca da inovação tecnológica para o etanol de segunda geração, sendo essa a principal contribuição deste artigo. Há escassez de estudos que comparam esforços de países na corrida pela busca de tecnologia de etanol de segunda geração. Alguns trabalhos com foco em inovação tecnológica para o setor possuem uma vertente histórica (SACHS, 2005; MACEDO, 2007); outros fazem uma abordagem geral (e não comparativa) do potencial brasileiro - ou a falta dele - para a continuidade da liderança tecnológica (LEITE; LEAL, 2007); ou ainda outros que avaliam políticas nacionais, como Jensen et al. (2010) nos EUA.

\section{Redes de inovação}

O conceito de redes de inovação, segundo Borgatti e Foster (2003), pode ser definido como um grupo de pessoas, equipes, organizações, entre outros atores, conectadas por um conjunto de laços que são os vínculos que podem ser: direcionados (conselho); indiretos; dicotômicos ou valorados (grau de amizade entre dois atores, por exemplo).

Elas também podem ser entendidas como articulações entre organizaçôes, que podem ser: várias empresas, universidades, agências regulatórias, etc. (RYCROFT; KASH, 2004), ou seja, trata-se de interação interorganizacional de atores heterogêneos, por meio de ações coletivas voltadas à inovação (PELLEGRIN et al., 2007), configurando-se como infraestruturas reais e virtuais (CARAYANNIS et al., 2006).

Freeman (1991) realizou uma síntese das pesquisas em redes de inovação e elegeu a definição de Imai e Baba (1989) como a mais interessante. Segundo esses autores, rede é um arranjo institucional básico para enfrentar a inovação sistêmica, podendo ser vista como uma forma de interpenetração do mercado e da organização, em que o relacionamento cooperativo entre as empresas é a chave para a formação de redes de inovação.

Nesse sentido, para Amato Neto (2000), as redes agregam diferentes funções e regulam a interdependência de sistemas complementares, possuindo três fatores 
determinantes para sua formação: a diferenciação, que estimula a inovação na rede sem exigir investimentos relevantes; a interdependência, que motiva a formação da rede e lhe confere unidade organizacional; e a flexibilidade no aspecto inovador, produtivo e organizacional, que permite à rede adaptação às mudanças do ambiente, gerando vantagem competitiva.

As redes de inovação surgem da cooperação dos atores para adquirir recursos que não poderiam ser obtidos individualmente (GUARAU, 2005). Assim, os agentes se articulam para criar, capturar e integrar as diferentes habilidades e o conhecimento necessário para desenvolver complexas tecnologias e introduzi-las no mercado (RYCROFT; KASH, 2004).

Tretyak (2001) também defende que a dependência mútua de troca e a existência de numerosas relações horizontais constituem o fator motivador para a criação de redes, levando empresas independentes e organizações autônomas especializadas e com objetivos próprios a fazerem parte de um sistema maior, com objetivos comuns, mas sem qualquer controle hierárquico.

Como benefícios advindos da formação de redes de inovação, Carayannis et al. (2006) destacam a contribuição para o crescimento do empreendedorismo tecnológico, a promoção da criatividade, a ampliação da capacidade de invenção e a aceleração do processo de inovação, em âmbito privado e/ou público.

São também consideradas vantagens da organização em rede a geração, promoção e difusão intensiva do conhecimento a fim de promover o desenvolvimento tecnológico (LEMOS, 1999; PYKA; KUPPERS, 2003), sendo esse formato o defendido por Lemos (1999) como o mais adequado para gerar o conhecimento que leva à inovação.

Conclui-se que a formação de redes voltadas para a inovação teve como principal fator motivacional a necessidade de desenvolvimento tecnológico sistêmico, o que no recente contexto é objetivo árduo e muitas vezes inviável economicamente para a organização atingir individualmente. Assim, por meio de geração e difusão compartilhada do conhecimento, acelera-se o processo inovativo dos membros da rede. Contudo, isso somente ocorrerá, de fato, se houver confiança mútua entre os agentes, devido ao compartilhamento de $\mathrm{P} \& \mathrm{D}$ e ao caráter estratégico da inovação para as organizações. Nesse sentido, o relacionamento é ponto-chave para que as redes de inovação aconteçam.

A Figura 1 sintetiza a dinâmica de redes de inovação, desde sua formação até os seus resultados, ou seja, a própria inovação. 


\section{FIGURA 1}

Redes de inovação: formação e conceitos básicos

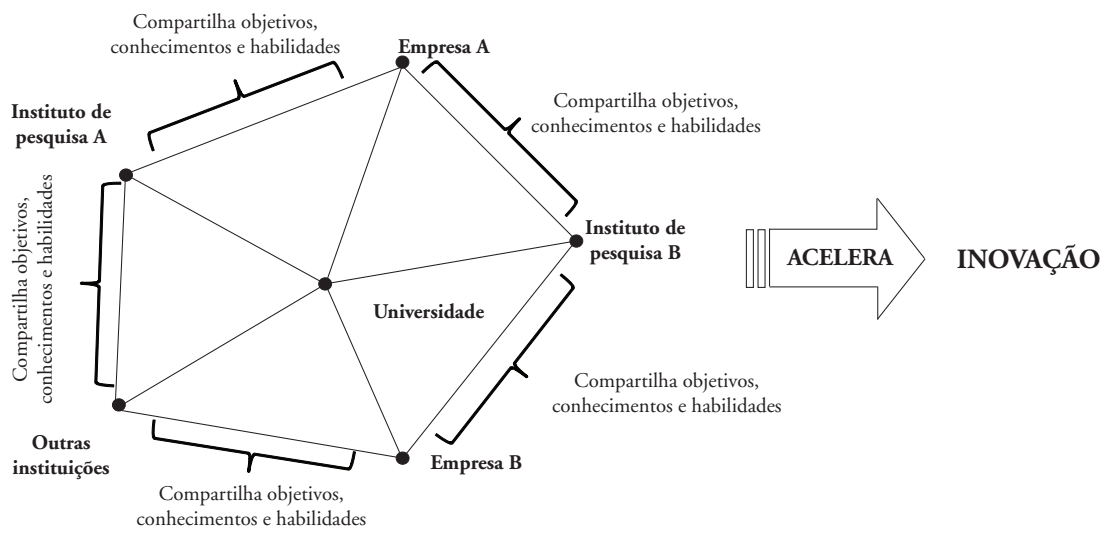

Como representada, a rede de inovação é uma estrutura formada por agentes com diferentes habilidades e conhecimentos, sendo que o trabalho em redes traz benefícios aos seus integrantes, com base na lógica da cooperação para desenvolvimento tecnológico. Dessa forma, os atores envolvidos conseguem compartilhar projetos complexos por meio da identificação das competências tecnológicas de cada um desses atores específicos e escolha de qual instituição tem as melhores condiçóes em termos tanto de pessoal qualificado quanto de infraestrutura para o desenvolvimento de uma etapa ou diversas etapas do trabalho.

Essa dinâmica permite a ampliação do número de especialistas dedicados à solução do problema, a redução do tempo de desenvolvimento e o compartilhamento dos custos e riscos. Assim, ao se constituir uma rede de inovação, seus integrantes estão, para aquele tema em particular, maximizando suas capacidades e reduzindo os riscos associados à motivação e o tempo de desenvolvimento. Uma vez que a rede baseia-se na parceria, esta só ocorrerá quando os atores perceberem que cooperativamente suas chances de sucesso se intensificam.

A formação das parcerias que envolvem empresas e pesquisadores advindos das ICT (instituições de ciência e tecnologia) que deverão suportar a construção de redes de inovação motiva as empresas em razão da

possibilidade de acesso à infraestrutura tecnológica, a qual abrange desde o acesso às instalaçôes universitárias, até os pesquisadores que ali trabalham, utilizando as 
instalaçôes de PUD de forma colaborativa, obtendo recursos governamentais de apoio à pesquisa e informaçôes de base tecnológica atualizadas. Para a empresa, a cooperação com a universidade surge como uma forma de suprir as suas carências em termos desta infraestrutura, contribuindo, assim, para melhorar a imagem da empresa, alçando-a ao patamar de organização inovadora, preocupada com tecnologia. (PORTO, 2006, p. 26).

No entanto, as dificuldades estão atreladas a diversos aspectos, entre os quais se destacam: a própria natureza da pesquisa, QUE, quanto mais próxima do conceito "pré-competitivo", maiores são as dificuldades de adesão ao risco tecnológico; a frequente necessidade por confidencialidade em relação aos resultados das pesquisas; os altos custos envolvidos em projetos de $\mathrm{P} \& \mathrm{D}$; a valorização técnica e comercial apenas para o que é realizado internamente, "suspeitando" das contribuiçõos de origem externa; a competição por consumidores; os aspectos culturais, inerentes aos empresários latino-americanos, resistentes à inovação e, em alguns casos, até à internacionalização; a falta de tempo por parte da empresa, devido à pressão dos negócios; e a falta de interesse na aquisição e utilização das tecnologias desenvolvidas por ICT, devido à escassez de informações sobre a produção destas instituições (PORTO, 2006).

Assim, após conseguir equalizar as motivaçôes e dificuldades para o surgimento das parcerias e, consequentemente, da rede de inovação, a mesma será constituída e operada conforme o modelo definido nos convênios e/ou contratos que a formalizaram. A estabilidade jurídica dos acordos também é um componente que interfere na longevidade e robustez da rede.

No caso das redes de inovação surgidas por meio de cooperação entre empresa, universidade e governo, as ações governamentais, como o bay-dohle americano e a lei de inovação brasileira, são os pontos de partida, a partir dos quais cada ator definirá suas premissas básicas e regras de operação, as quais determinarão os fatores de diferenciação, interdependência e flexibilidade da rede.

\section{Metodologia}

A presente pesquisa pode ser classificada como exploratória, tendo por objetivo "conhecer as características de um fenômeno para procurar, posteriormente, explicaçôes das causas e consequências de dito fenômeno" (RICHARDSON, 1999, p.326), uma vez que se trata de um movimento recente, com poucos resultados sistematizados e publicados. 
$\mathrm{O}$ desenvolvimento da pesquisa ocorreu em duas etapas. Na primeira, com o propósito de verificar a situação do Brasil em relação aos outros países no que se refere à realização de $\mathrm{P} \& \mathrm{D}$ de novas rotas tecnológicas para a produção de etanol celulósico, que tornem sua produção viável economicamente em escala comercial, foram escolhidos dois indicadores: o levantamento bibliométrico e o de pedidos de patentes. A escolha desses indicadores justifica-se pela sua abrangência, uma vez que a esfera "Ciência" foi explorada por meio de pesquisa a respeito de trabalhos acadêmicos com o tema "etanol celulósico" na base de dados multidisciplinar Web of Science. A palavra-chave utilizada em tal procedimento foi "bioethanolhydrolysis".

Já a esfera "Tecnologia" foi trabalhada por meio da realização de uma busca no banco de dados Esp@cenet. Este é um serviço de busca, online e gratuito, desenvolvido pelo escritório europeu de patentes e pela organização europeia de patentes, que concentra os pedidos de patentes efetuados em diversos escritórios, entre eles Brasil, EUA e União Europeia. Nesse procedimento foram utilizadas a palavra-chave "bioethanolhydrolysis" e combinações de termos relacionados, tais como "enzymatichydrolysisandlignocellulosic", "ethanollignocellulosicandenzyme" e "acidlignocellulosicandhydrolysis", para que o banco de dados fosse amplamente explorado. Todas as buscas ocorreram no Advanced Search, selecionando a base de dados worldwide, que aglomera os bancos de patentes de mais de 80 países e as palavras-chave foram digitadas no campo "Keyword(s) in titleor abstract".

$\mathrm{Na}$ segunda etapa, com o intuito de comparar os programas brasileiros de P\&D de etanol celulósico, selecionou-se o país de maior destaque nas esferas C\&T, evidenciado na primeira parte do estudo. Para tal, utilizaram-se as informações disponíveis $^{3}$ no site oficial de três programas de incentivo a P\&D do etanol celulósico: o programa bioenergia (Bioen), o laboratório nacional de ciência e tecnologia do bioetanol (CTBE), ambos do Brasil, e o Biomass Program, dos Estados Unidos.

\section{Resultados e discussão}

\subsection{Esforços para desenvolvimento tecnológico}

Na busca realizada no Esp@cenet, foram encontrados 95 pedidos de patentes, cujo conteúdo a ser patenteado referia-se a etanol celulósico, sendo que o pedido mais recente era de 2010 e o mais antigo de 1981. Já a coleta de dados bibliométricos resultou em 403 artigos publicados entre 1990, ano da primeira publicação, e

3 Consulta a relatórios e documentos oficiais. 
2010. Contudo, os dados do Gráfico 1 mostram um aumento considerável dos pedidos de patentes e de artigos nos últimos anos, indicando que se trata de um assunto contemporâneo e que a corrida pela descoberta de novas rotas tecnológicas de produção de etanol em escala comercial elevou o número de estudos nessa área.

GRÁFICO 1

Pedidos de patentes e artigos publicados relacionadas à produção de etanol celulósico 1981-junho 2010
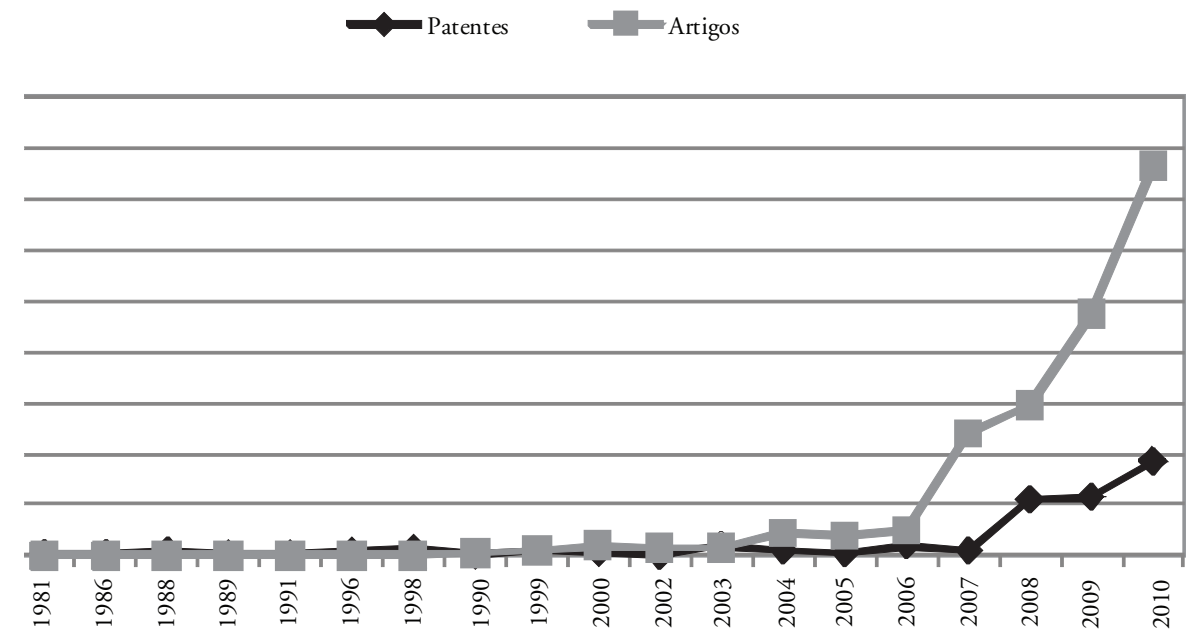

Fonte: Elaboração a partir de dados do Esp@cenet e Web of Science

Com relação à origem da patente, foi considerado o país onde o requerente (pessoa física, jurídica ou órgão governamental) está ou estava instalado no momento da solicitação da patente. Essa informação é de suma importância para o trabalho, pois identifica quais os países onde a P\&D que definira as rotas tecnológicas do etanol está mais desenvolvida (Tabelas 1 e 2).Vale ressaltar que três pedidos foram realizados por instituições de países diferentes, sendo considerado o país do primeiro aplicante.

Já no caso dos artigos científicos, foi considerado o país da instituição de pesquisa a qual o pesquisador pertencia, informado na publicação. Dos 403 artigos cujo assunto estava relacionado a etanol celulósico, observaram-se desde novos métodos de produção até revisões da literatura sobre as pesquisas que envolvem essa temática e o impacto desta inovação na economia local e mundial. Vale ressaltar que, das publicaçôes levantadas, 49 artigos envolviam mais de um país, sendo considerada, para contagem, a nação da instituição do primeiro autor. 
TABELA 1

Pedidos de patentes, segundo país de origem do primeiro requisitante $1981-2010$

\begin{tabular}{l|c|c}
\hline \multicolumn{1}{c|}{ País } & N. absolutos & $\%$ \\
\hline Estados Unidos & 32 & 33,7 \\
Canadá & 20 & 21,1 \\
França & 7 & 7,4 \\
Brasil & 6 & 6,3 \\
República Checa & 5 & 5,3 \\
Dinamarca & 4 & 4,2 \\
China & 4 & 4,2 \\
Japão & 4 & 4,2 \\
Alemanha & 2 & 2,1 \\
Índia & 2 & 2,1 \\
Outros & 9 & 9,5 \\
\hline
\end{tabular}

Fonte: Elaboração a partir de dados do Espacenet.

\section{TABELA 2}

Artigos publicados referentes ao tema etanol celulósico, segundo país da instituição de pesquisa

$1981-2010$

\begin{tabular}{lccc}
\hline \multicolumn{1}{c|}{ País } & N. absolutos & $\%$ \\
\hline Estados Unidos & 69 & 17,12 \\
Suécia & 30 & 7,44 \\
Japão & 29 & 7,20 \\
Dinamarca & 28 & 6,95 \\
Espanha & 23 & 5,71 \\
China & 23 & 5,71 \\
Brasil & 17 & $\mathbf{4 , 2 2}$ \\
Canadá & 16 & 3,97 \\
Índia & 15 & 3,72 \\
Inglaterra & 14 & 3,47 \\
Coréia do Sul & 14 & 3,47 \\
Turquia & 11 & 2,73 \\
Outros & 114 & 28,29 \\
\hline
\end{tabular}

Fonte: Elaboração a partir de dados do Web of Science. 
Observando-se as Tabelas 1 e 2, parece haver maior concentração de esforços dos países na esfera Ciência, sendo registradas 46 diferentes nações contra 18 da esfera Tecnológica. Em ambas, identifica-se claramente a liderança dos Estados Unidos, o que indica, que além de produzir conhecimento nessa área, o país também procura desenvolver tecnologia e protegê-la, dada a importância estratégica do seu domínio para futura exploração comercial.

Quanto ao objetivo principal da pesquisa, que era verificar a posição brasileira em ambas as esferas C\&T, nota-se que a participação do Brasil é pequena tanto na produção científica, com apenas 4,22\% dos trabalhos publicados, como na solicitação de patente internacional por meio do PCT (6,3\%), apesar de o etanol ter representado $5,7 \%^{4}$ da matriz energética nacional em 2009 e seu consumo ter registrado aumento de 7,1\% em relação a 2008 (BRASIL, 2010). Nos Estados Unidos, a participação dos biocombustíveis está em aproximadamente $1,37 \%$ da matriz energética (EIA, 2010).

Diante desse cenário, indicado pelos inexpressivos resultados brasileiros tanto na esfera científica como na tecnológica, e tendo em vista que o etanol celulósico é foco de política pública no Brasil e também nos Estados Unidos, país de destaque em ambas as esferas, optou-se por comparar os programas de desenvolvimento de P\&D em etanol celulósico desses dois países, a fim de compreender os resultados científicos e tecnológicos discutidos nessa seção. No Brasil, trabalhou-se com dois programas: um de âmbito estadual, o programa Bioenergia (Bioen); e outro de nível federal, o Laboratório Nacional de Ciência e Tecnologia do Bioetanol (CTBE). Para os Estados Unidos, foi analisado o Biomass Program. Os três são os mais desenvolvidos em seus países e têm como característica principal a estruturação em rede, formada a partir do compartilhamento de conhecimento, recursos e infraestrutura entre empresas, institutos de pesquisa, universidade, órgãos de fomento e governo.

\subsection{Redes para desenvolvimento tecnológico}

\subsubsection{Fapesp/Bioen}

O programa Bioenergia (Bioen) foi criado em 2008, pela Fundação de Amparo à Pesquisa do Estado de São Paulo (Fapesp), com o objetivo de estimular e articular $\mathrm{P} \& \mathrm{D}$ relacionado à produção de etanol, utilizando a infraestrutura tanto de laboratórios das universidades e institutos de pesquisa quanto de indústrias. Para tal, o

4 No mesmo período a participação da gasolina foi de 7,1\%, com uma taxa de crescimento de 0,9\% (BRASIL, 2010). 
programa foi estruturado em cinco divisões: biomassa para bioenergia, concentrada em cana-de-açúcar, incluindo genoma, bioquímica, biologia celular, fisiologia, melhoramento genético e tecnologias de cultivo de cana; processo de fabricação de biocombustíveis, cujos estudos estão voltados para o aumento da produtividade de etanol por tonelada de cana e redução do uso de água, energia e impactos ambientais do processo - é nessa linha que se encontram os estudos relacionados ao etanol celulósico; biorrefinarias e alcooquímica, focada no desenvolvimento de produtos de alto valor agregado a partir de matérias-primas renováveis (derivados químicos e petroquímica); aplicaçóes do etanol para motores automotivos; e pesquisa sobre os impactos socioeconômicos, ambientais e uso da terra.

O programa é composto por três empresas, seis universidades com seus diversos departamentos, quatro institutos, um centro de pesquisa, uma agência e uma associação de tecnologia (Figura 2), todos localizados no Estado de São Paulo. Seu funcionamento ocorre por meio de chamadas, organizadas pela Fapesp que seleciona projetos de pesquisa que atendam às necessidades de $\mathrm{P} \& \mathrm{D}$ estabelecidas nas cinco divisões do Bioen e também aos convênios estabelecidos com as organizações. Entre as três empresas participantes do programa, duas firmaram convênio para o desenvolvimento de pesquisas maiores relacionadas à produção de etanol celulósico: a Dedini S/A Indústria de Base; e a Oxiteno S/A - Indústria e Comércio.

No caso da Dedini, o convênio foi firmado por cinco anos, após uma primeira parceria entre a empresa e a Fapesp via o Programa de Inovação Tecnológica - Pite (em que também estavam envolvidos o Centro de Tecnologia Canavieira - CTC, Copersucar e Unicamp). O valor total do acordo é de R $\$ 100.000 .000,00$, sendo que cada uma das partes é responsável por $50 \%$ do montante. As pesquisas desenvolvidas dentro do acordo são realizadas por meio de chamadas divulgadas pela Fapesp e as propostas são escolhidas pela fundação juntamente com o comitê gestor da cooperação (composto por dois integrantes de cada parte) e visam atender principalmente às demandas do projeto Dedini Hidrólise Rápida - DHR. Com relação à propriedade intelectual das inovações geradas, estas são acordadas em termos de convênio entre a Dedini e as instituiçóes de pesquisa, podendo ter ou não a participação da Fapesp.

O convênio entre a Oxiteno e a Fapesp também possui duração de cinco anos e poderá chegar a um valor total de $\mathrm{R} \$$ 6.000.000,00 sendo que metade do valor é de responsabilidade da empresa e a outra metade será dividida igualmente entre a Fapesp e o BNDES, por meio de um convênio entre essas duas instituições. Assim como no outro convênio, as propostas serão divulgadas pela Fapesp, mas selecio- 
nadas de acordo com os critérios do Pite. Para a divisão da propriedade intelectual as mesmas regras foram estabelecidas.

No tocante à participação das universidades e instituições de pesquisa, até junho de 2010, 47 projetos (concluídos ou não) foram financiados por recursos do Bioen, sendo 41 ligados ao etanol de segunda geração. Vale ressaltar que, para participar das chamadas realizadas pela Fapesp, a instituição de pesquisa deve estar localizada no Estado de São Paulo, contudo, nota-se a presença de diversas instituiçōes federais e estaduais de outras unidades federativas, como colaboradoras nos projetos participantes do Bioen ligados às instituiçóes paulistas. $\mathrm{O}$ trabalho conjunto desses atores dá origem à rede de inovação descrita na Figura 2.

FIGURA 2

Rede de inovação criada pelo projeto Bioen-Fapesp

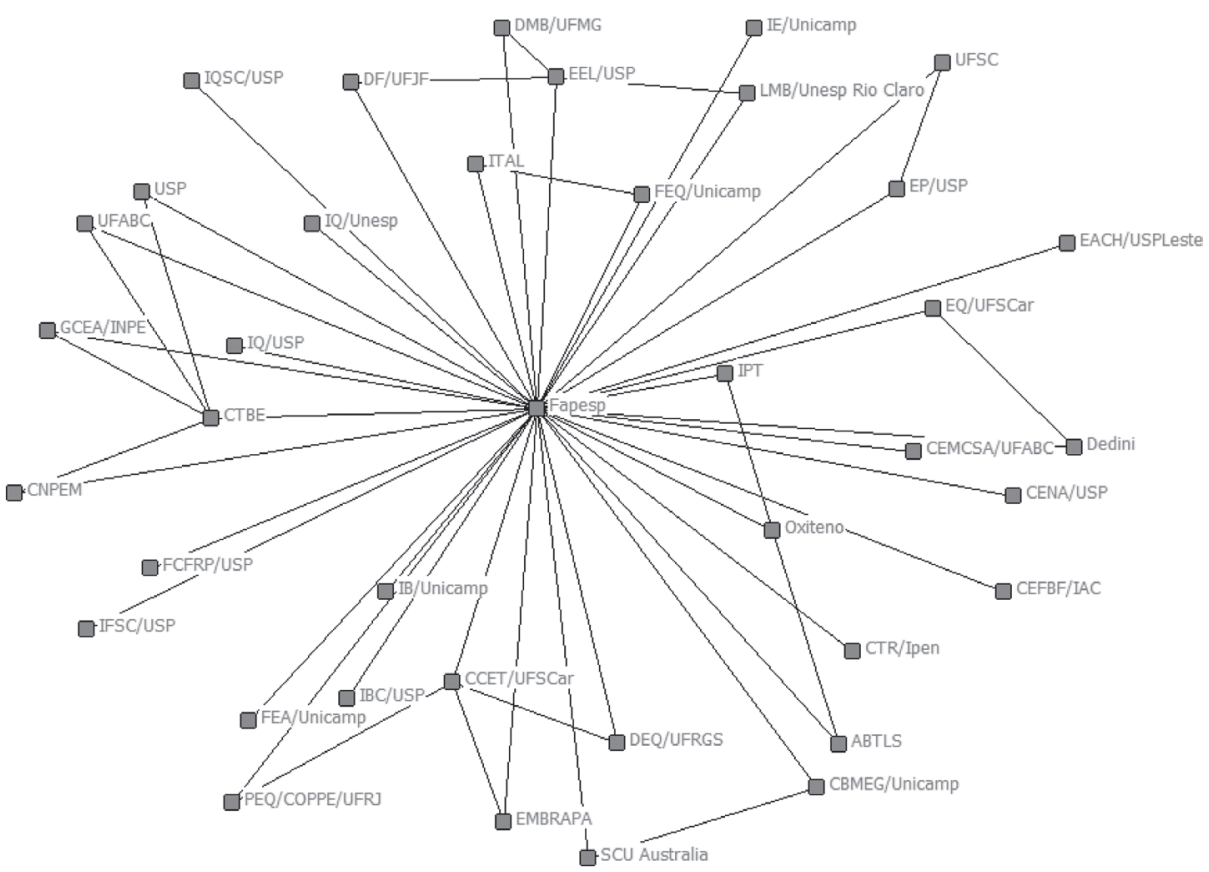

A Figura 2 apresenta a rede formada pelos projetos que integram o Bioen, tendo a Fapesp como o centro (egorede), pois a instituição participa de todos os convênios. A rede é formada por 39 nós e 56 ligações, indicando uma rede de inovação com baixa densidade $(0,119)$ e elevada fragmentação $(0,895)$. A rede apresenta buracos estruturais em razão da baixa densidade e elevada fragmentação. 


\subsubsection{Laboratório Nacional de Ciência e Tecnologia do Bioetanol (CTBE)}

O CTBE é um laboratório nacional, vinculado ao Ministério de Ciência e Tecnologia, que foi criado em janeiro de 2010 para viabilizar a intenção brasileira de substituir $10 \%$ de toda a gasolina consumida no mundo até $2025 .{ }^{5}$ As atividades do CTBE estão estruturadas em dois pilares: a pesquisa externa e a interna.

Os estudos internos são realizados por pesquisadores do laboratório e divididos em três linhas: estudo da conversão de energia solar em biomassa, ou seja, a obtenção dos açúcares contidos na parede celular da cana; estudo da conversão de biomassa em etanol, cujo principal objetivo é obter conhecimento para a concepção de usinas integradas, que realizem a disponibilização do material lignocelulósico, hidrólise e fermentação; e estudo da conversão de etanol em energia mecânica (pesquisa em motores movidos a etanol).

A pesquisa externa ocorrerá via cooperação com empresas privadas, universidade e institutos de pesquisa nacionais e também internacionais, como, por exemplo, o convênio firmado entre o centro e o consórcio chileno Bioenercel S.A. para o desenvolvimento conjunto da tecnologia de etanol celulósico de cana e madeira, respectivamente. Estes ocorrerão por meio da disponibilização de uma planta-piloto, que permite a produção de etanol em escala semi-industrial; ou seja, diferentemente dos outros programas analisados nesse artigo, o fomento à pesquisa não acontece por meio da disponibilização de recursos financeiros, mas sim pelo compartilhamento de estrutura.

Essa cooperação visa acelerar o processo de desenvolvimento de produto e processo, pois dificilmente as referidas organizações disporiam de estrutura e recursos suficientes para produzir em escala comercial o etanol desenvolvido em laboratório. Consequentemente, a transferência de tecnologia promove o aceleramento e a melhoria do P\&D realizado internamente pelo laboratório.

O laboratório foi inaugurado em janeiro de 2010, com previsão para final de 2011 de finalização da construção da planta-piloto para desenvolvimento de processos (PPDP) e os últimos laboratórios. Ele possui parcerias com Embrapa, Unicamp, Lund University - Suécia, Laboratório Nacional de Energia Renovável dos Estados Unidos - NREL, Imperial College London, Universidade de Caxias do Sul, Rhodia,

5 Essa meta faz parte de um objetivo mundial de substituir $25 \%$ do petróleo utilizado mundialmente por biocombustíveis. Este assunto é tema de discussão atual, como, por exemplo, a realizada pela Convenção Latino-Americana do projeto Global Sustainable Bioenergy (GSB) em 2010, uma vez que se trata de um objetivo de longo prazo. 
Novozymes, Pedra Agroindustrial S/A, etc. As estruturas de governança a serem adotadas para gerenciar o relacionamento entre os centros de pesquisa, empresas privadas e o laboratório encontram-se em processo de formulação. Basicamente a rede de inovação que será gerada pelas operações do CTBE está descrita na Figura 3 , não sendo possível um maior detalhamento como o realizado para os outros programas devido à rede ainda estar sendo formada.

FIGURA 3

Modelo da rede de inovação criada pelo CTBE

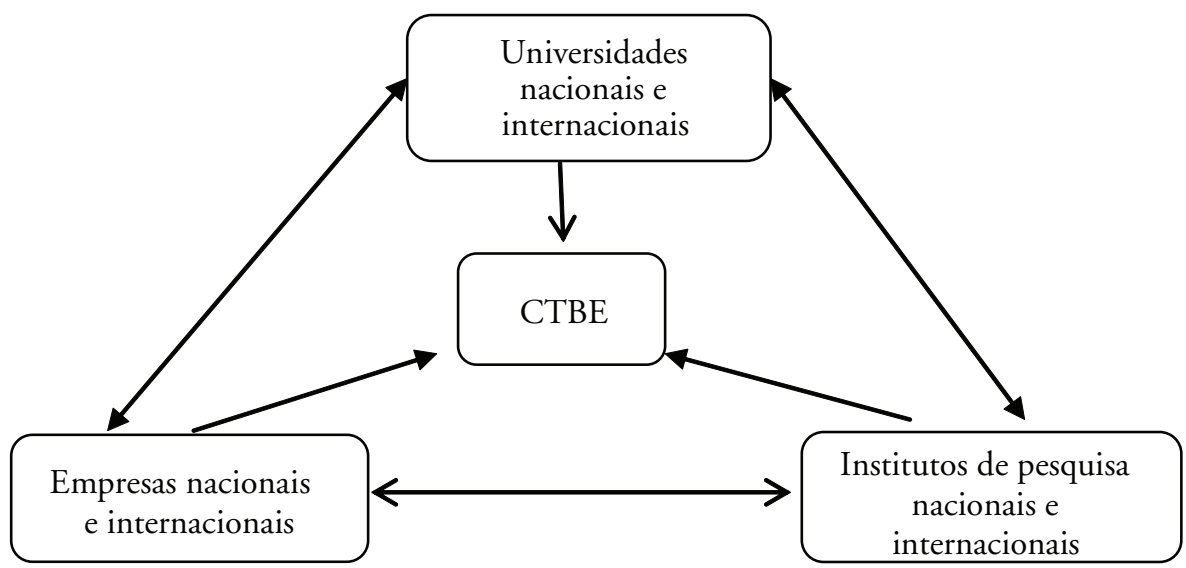

Além dos dois programas discutidos anteriormente, ocorrem no Brasil ações isoladas, como a atuação das universidades na corrida pelo desenvolvimento em escala comercial do etanol celulósico, com quatro solicitações de patentes, além da publicação de artigos. Essas ações acontecem isoladamente ou por meio de cooperação entre universidade e empresa, identificada pela cotitularidade dos pedidos de patentes.

Paralelamente, algumas empresas brasileiras ou com subsidiárias instaladas no país também possuem $\mathrm{P} \& \mathrm{D}$ em etanol celulósico, como a Petrobrás e a Shell respectivamente. Embora existam esses projetos, as organizações que hoje ocupam espaço de destaque no fornecimento de etanol de primeira geração estão praticamente fora desta disputa até a presente data, uma vez que ainda não investem nesta rota tecnológica. 


\subsubsection{Biomass Program}

Os Estados Unidos consideram estratégico reduzir sua dependência por combustíveis fósseis, principalmente pela necessidade de abastecimento por meio de fontes externas da sua demanda. Para tal, a partir da década de 2000, o país intensificou recursos e esforços a fim de alcançar esse objetivo de longo prazo. Nesse sentido, em 2007, foi criado o Programa Biomassa, sob responsabilidade do departamento de energia estadunidense, que compilou e reforçou algumas das iniciativas existentes, além de desenvolver outras. O objetivo é articular parcerias com a indústria, a academia e os laboratórios nacionais, para apoiar pesquisa e desenvolvimento que visem a transformação da biomassa em bioenergia, a fim de que, até 2012, o etanol celulósico possa ser produzido em escala comercial a um preço competitivo.

Para tal, com recursos governamentais e de outras fontes, são financiados projetos e atividades de pesquisa, desenvolvimento e implementação, enviados por universidades, laboratórios de pesquisa e empresas privadas, em resposta às chamadas públicas divulgadas pelo programa. No caso das indústrias, o custo de P\&D é dividido, variando de $20 \%$ nos estágios mais precoces a no mínimo $50 \%$, quando os resultados da pesquisa se encontram próximos do desenvolvimento comercial.

A estrutura de P\&D do programa está dividida em cinco linhas: P\&D na interface de abastecimento de biomassa, cujo objetivo é desenvolver tecnologias sustentáveis capazes de suprir as biorrefinarias com biomassa lignocelulósica de baixo custo e alto volume; P\&D para a plataforma de açúcares, em que se busca a obtenção do açúcar por meio da quebra da biomassa via tratamento químico ou biológico de fontes vegetais; P\&D para a plataforma termoquímica, buscando converter a biomassa ou resíduos de biomassa derivados da biorrefinaria em intermediários como gás sintético, que podem ser usados diretamente como combustíveis crus ou produtos, ou podem ser subsequentemente refinados para obtenção de combustíveis ou produtos intercambiáveis com commodities comerciais já existentes - como óleos, gasolina, diesel, gás natural sintético e hidrogênio de alta pureza; P\&D de produtos, o que corresponde à transformação dos produtos da plataforma de açúcares em produtos finais, com alto volume e valor agregado; e P\&D biorrefinarias integradas, uma divisão conduzida exclusivamente pela 
indústria, sendo um complemento das anteriores, pois visa a produção em larga escala das tecnologias desenvolvidas anteriormente.

A rede formada pelo Biomass Program envolve as esferas pública e privada presentes nos Estados Unidos e em outros países, ocorrendo tanto o contato direto do programa com os agentes isoladamente, quanto uma cooperação entre estes para atender a uma necessidade do programa divulgada em chamadas públicas.

Segundo relatórios disponíveis no site do programa, até o momento 130 projetos foram ou estão sendo financiados por recursos do Biomass Program, envolvendo um total de 202 agentes, sendo que 101 encontram-se envolvidos em pesquisas relacionadas ao etanol celulósico. São institutos de pesquisa, universidades, centros de ensino e empresas privadas, localizados principalmente nos Estados Unidos, mas também em outros lugares do mundo.

Ao se analisar o tipo de agente, nota-se um maior envolvimento da iniciativa privada, com participação de aproximadamente $60 \%$ dos agentes, seguida pelas universidades, que respondem por $22 \%$ dos participantes, e pelas instituições ligadas ao governo dos Estados Unidos ou de outros países, com 18\% dos agentes. Essa maior participação pode ser justificada pelos projetos de biorrefinaria, até o momento desenvolvidos apenas por indústrias.

A Figura 4 apresenta o desenho da rede criada pelo Biomass Program, que apresenta o departamento de energia dos Estados Unidos como centro da rede, uma vez que este participa de todos os convênios como financiador.

É uma rede composta por 322 ligações e 219 nós, atingindo uma densidade de $(0,035)$ com uma fragmentação de $(0,522)$, apresentando-se como a mais robusta das três redes estudadas. Conforme a Figura 4, observa-se a característica de rede egocêntrica, com as instituiçôes de pesquisa ocupando a parte central e os atores empresariais na periferia da rede. Assim os atores que irradiam as parcerias são principalmente ICT.

Dos 130 projetos listados, $21 \%$ são de plantas-piloto de biorrefinarias, o que pode indicar um alto grau de desenvolvimento do etanol celulósico em escala semi-industrial e industrial, um diferencial em relação a outros países, inclusive o Brasil, onde há apenas uma planta-piloto para desenvolvimento de processos em uma empresa privada (Bioen/Dedini/Fapesp) e outra em fase de construção no CTBE. 


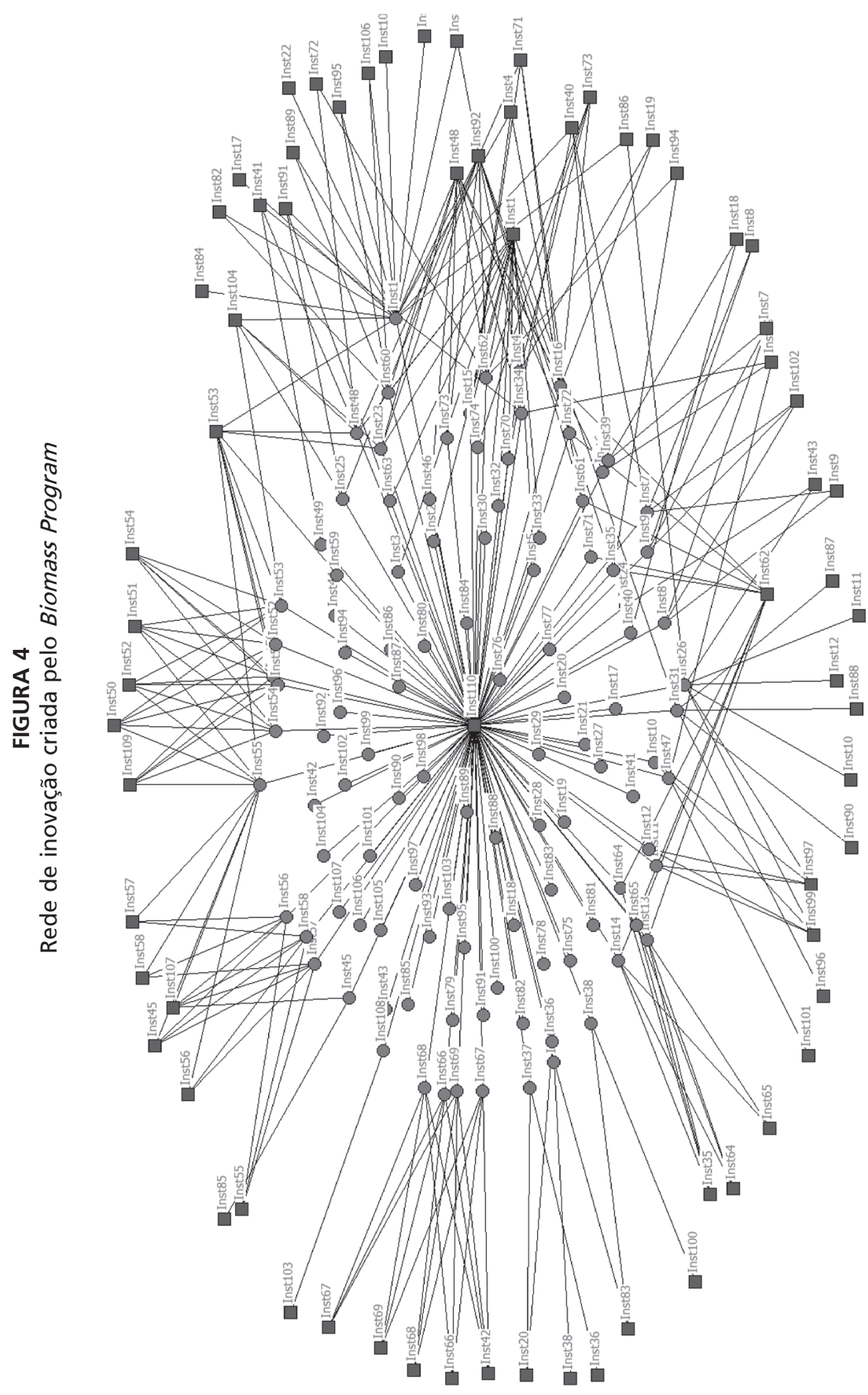




\subsubsection{Benefícios capturados pelas três redes de inovação}

A formação das três redes de inovação a partir dos programas Bioen, CTBE e Biomass Program propiciou o aferimento de alguns dos benefícios listados na literatura, quando da ocorrência de redes de inovação (Quadro 1). Alguns deles são comuns às três iniciativas e outros são frutos do esforço particular de um dos programas.

\section{QUADRO 1}

Benefícios da formação de redes de inovação, segundo os programas Bioen, CTBE e Biomass Program

\begin{tabular}{l|c|c|c}
\hline \multicolumn{1}{c|}{ Benefícios oriundos de redes de inovação } & Bioen & CTBE & $\begin{array}{c}\text { Biomass } \\
\text { Program }\end{array}$ \\
\hline $\begin{array}{l}\text { Promover a criatividade, ampliar a capacidade de invenção e } \\
\text { acelerar o processo de inovação (CARAYANNIS et al., 2006) }\end{array}$ & $\mathrm{x}$ & $\mathrm{x}$ & $\mathrm{x}$ \\
$\begin{array}{l}\text { Criar, capturar e integrar diferentes habilidades e } \\
\text { conhecimentos para desenvolver complexas tecnologias }\end{array}$ & $\mathrm{x}$ & & $\mathrm{x}$ \\
(RYCROFT; KASH, 2004) & $\mathrm{x}$ & $\mathrm{x}$ & $\mathrm{x}$ \\
$\begin{array}{l}\text { Gerar e difundir conhecimento para promover o } \\
\text { desenvolvimento tecnológico (PYKA; KUPPERS, 2003) }\end{array}$ & & $\mathrm{x}$ \\
$\begin{array}{l}\text { Flexibilidade no aspecto inovador, produtivo e organizacional } \\
\text { (AMATO NETO, 2000) }\end{array}$ & & $\mathrm{x}$ & $\mathrm{x}$ \\
Inovação estimulada pela diferenciação (AMATO NETO, 2000) & $\mathrm{x}$ & $\mathrm{x}$ & $\mathrm{x}$ \\
Aprendizado intensivo e geração de conhecimento e de \\
inovaçães (LEMOS, 1999)
\end{tabular}

No tocante à aquisição de infraestrutura, o formato do programa CTBE diferencia-se dos outros analisados, uma vez que a grande motivação para a formação da rede é o compartilhamento da planta-piloto, viabilizando a execução de pesquisas, iniciadas em laboratório, nos seus estágios mais avançados (escala industrial). Dessa forma, nota-se claramente que, por meio da rede, foi possível adquirir infraestrutura (planta-piloto) que dificilmente poderia ocorrer, considerando-se os agentes isoladamente. $\mathrm{Na}$ rede criada pelo Bioen é possível a obtenção de recursos, mas não necessariamente isso pode ser listado como um benefício que permite obter infraestrutura. No caso do Biomass Program, apesar dessa rede contemplar seis biorrefinarias, elas são propriedades particulares que recebem recursos do programa, 
por meio de parceria estabelecida, não sendo um benefício exclusivo da formação da rede, pois poderia ocorrer sem a existência da mesma, sendo a contrapartida das empresas.

Produzir etanol celulósico em escala comercial competitivamente é objetivo dos três programas, portanto, promover a criatividade, ampliar a capacidade de invenção e acelerar o processo de inovação são benefícios verificados nas três redes de inovação, pois basicamente foram formadas para tal. Esses aspectos estão mais presentes, no Bioen, pelo relacionamento entre os grupos de pesquisa sobre biocombustíveis; no CTBE, por meio do compartilhamento de uma planta-piloto; e, no Biomass Program, via a meta estabelecida, para até 2012 desenvolver a tecnologia que viabilize a produção de etanol celulósico comercialmente, o que motiva os componentes dessa rede a acelerarem o processo de inovação.

Da mesma forma, criar, capturar e integrar diferentes habilidades e conhecimentos para desenvolver complexas tecnologias constitui a base para a criação das redes estudadas. A fim de obter esses benefícios, tanto o Bioen quanto o Programa Biomassa foram estruturados, sendo que seu funcionamento consiste basicamente em buscar alternativas de produção do etanol celulósico comercialmente, a partir de pesquisas realizadas por diferentes universidades, empresas privadas e institutos de pesquisa. No CTBE a cooperação ocorre por meio do compartilhamento da planta-piloto, diferentemente dos outros programas estudados. Contudo, apesar de não haver o desenvolvimento de pesquisas conjuntas entre os agentes, espera-se que ocorra, por parte dos pesquisadores do CTBE, um aproveitamento das experiências de outros estudos realizados em suas imediações.

Assim, por meio da exploração dos diferentes enfoques e estudos, orientados por um objetivo comum, nota-se que a formação das três redes, como apontado pela literatura, utiliza a diferenciação para estimular a inovação na rede, minimizando a exigência de investimentos relevantes e promovendo o aprendizado intensivo e a geração de conhecimento e consequentemente de inovaçóes, expressos por meio da publicação de artigos e pedidos de patentes.

O Biomass Program parece ser o único ao auferir o benefício da flexibilidade nos três aspecto apontados por Amato Neto (2000), pois admite que tanto instituições estadunidenses como de outros países participem de suas chamadas, permitindo que os processos estudados nos Estados Unidos para a fabricação do etanol celulósico possam ser comparados e incrementados com métodos desenvolvidos em outras regiōes, o que facilita a agregação de conhecimento de fontes externas e auxilia o acesso a esse e à informação. 
Nesse sentido, o CTBE pretende estabelecer um relacionamento parecido, contudo, suas formas ainda se encontram em elaboração. O Bioen, por ser um programa desenvolvido por uma agência de fomento estadual, tem o menor grau de agregação de conhecimento externo, pois a participação de empresas, universidades e centros de pesquisa fica restrita à localização territorial, embora instituições de pesquisa de outras regióes possam atuar como colaboradoras no programa. Vale destacar que o CTBE é integrante da rede Bioen e desenvolve dois projetos nessa estrutura, ocorrendo uma integração entre redes de inovação.

Por fim, nota-se que as três redes estudadas facilitam a cooperação entre Estado, universidade e indústria, integrando diferentes tipos de conhecimento. Nos casos do Bioen e do Programa Biomassa, elas têm como motivação o atendimento às chamadas públicas realizadas, e no CTBE, ainda que em menor grau, poderá ocorrer devido ao compartilhamento de infraestrutura. Essa integração, segundo a literatura, é de grande importância para a aceleração do processo inovativo.

Vale ressaltar que, embora não se possa afirmar diretamente que nas redes estudadas houve a promoção do empreendedorismo tecnológico, benefício advindo da formação de redes de inovação, é possível que, na cadeia secundária, seja no assessoramento às organizações e/ou universidades participantes da rede, essa vantagem possa ter sido auferida.

\section{Considerações finais}

Ao se analisarem os dados de C\&T do Brasil e dos Estados Unidos e posteriormente comparar a estrutura dos principais programas de $\mathrm{P} \& \mathrm{D}$ em etanol celulósico dos dois países, verifica-se um considerável atraso brasileiro, a começar pelo início dos programas, dado que uma iniciativa articulada do governo federal só aconteceu em 2010, por meio da criação do CTBE, que, em termos de rede, possui uma estrutura diferenciada dos outros programas, pois o principal motivador de sua formação não é o recurso financeiro para o desenvolvimento de $\mathrm{P} \& \mathrm{D}$, mas sim o compartilhamento de infraestrutura. Dessa forma, torna-se possível a transferência de conhecimento entre os agentes que utilizaram a planta-piloto e o próprio CTBE.

O programa Bioen promovido pela Fapesp mostra ser a rede de inovação mais avançada em relação às outras desenvolvidas no Brasil, possuindo linhas 
direcionadoras dos esforços de P\&D e política de acesso ao programa definidas. Entretanto, tem como limitação a abrangência estadual, que restringe a expansão da rede e a obtenção de alguns dos benefícios oriundos da formação desse tipo de estrutura.

O Biomass Program nos EUA tem se mostrado mais eficaz no sentido de promover a formação de redes entre universidades, empresas e instituições que compartilham equipamentos e conhecimento, a fim de atender aos objetivos do programa estabelecidos nas chamadas. Esse desempenho deve-se, principalmente, à flexibilidade do programa com relação à origem dos integrantes da rede, o que permite ao país um maior conhecimento dos processos utilizados em outras nações, fundamental nesse momento, pois a descoberta e a aceleração do(s) processo(s) que permita $(m)$ a fabricação do etanol celulósico em escala comercial podem significar o domínio tecnológico daquele país.

Outro fator de destaque referente ao Programa Biomassa é que este constitui uma das iniciativas desenvolvidas pelo governo estadunidense que visa $P \& D$ de outras fontes energéticas, possuindo, para tal, um departamento que coordena esses esforços. Em comparação com os programas brasileiros, segundo os relatórios disponibilizados, ele dispôs de maior quantia de recurso financeiro, o que pode justificar, em parte, a posição de destaque dos Estados Unidos verificada a partir da análise dos indicadores de C\&T.

Conclui-se que a liderança dos Estados Unidos, tanto em artigos publicados como em solicitações de patentes, pode ser justificada pelo seu efetivo esforço em iniciar, articular e investir em P\&D de etanol celulósico, entre outras fontes. Nesse sentido, enquanto para os Estados Unidos a busca pela redução da dependência por combustíveis estrangeiros e, consequentemente, pelo desenvolvimento de outras rotas tecnológicas é estratégia nacional, para o Brasil, o domínio da tecnologia de produção do etanol celulósico significa a manutenção de uma posição de liderança ocupada atualmente pelo setor sucroenergético. Sob essa ótica, apesar de possuir papel fundamental nesse processo, o Estado ainda tem atuado de forma setorial e regional.

Por fim, nota-se que no Brasil ainda é baixo o envolvimento da iniciativa privada nas redes de P\&D do etanol celulósico, sendo duas empresas até o momento participantes, contra 117 nos Estados Unidos, o que reduz demasiadamente as chances de uma organização brasileira dominar essa nova rota tecnológica. 


\section{Referências bibliográficas}

AMATO NETO, J. Redes de cooperação produtiva e clusters regionais: oportunidades para as pequenas e médias empresas. São Paulo: Atlas, 2000.

ANFAVEA. Estatísticas. Disponível em: <http://www.anfavea.com.br/tabelas.html>. Acesso em: 08 jun. 2010.

BRASIL. Ministério de Minas e Energia (MME). Balanço Energético Nacional (BEN) 2009: ano base 2008 - relatório final. Rio de Janeiro: MME/EPE, 2009.

- Ministério de Minas e Energia (MME). Balanço Energético Nacional (BEN) 2010: ano base 2009 - dados preliminares. Rio de Janeiro: MME/EPE, 2010.

BIOMASS PROGRAM. Project FactSheets. U.S. department of energy. Disponível em: <http://www1.eere.energy.gov/biomass/factsheets.html>. Acesso em: jun. 2010.

BORGATTI S. P.; FOSTER, P. C. The network paradigm in organizational research: a review and typology. Journal of Management, v. 29, p. 991-1013, 2003.

FAPESP. Brazilian research on bioenergy. São Paulo: Fundação de Amparo a Pesquisa do Estado de São Paulo, jun. 2010.

CARAYANNIS et al. Technological learning for entrepreneurial Development. International. Techovation, n. 26, p. 419-443, 2006.

CARDONA, C. A.; QUINTERO; J. A.; PAZ, I. C. Production of bioethanol from sugarcane bagasse: status and perspectives. Bioresource Technology, n. 101, p. 4754-4766, 2010.

CTBE. Laboratório Nacional de Ciência e Tecnologia do Bioetanol. Disponível em: <http:// www.bioetanol.org.br/>. Acesso em: jul. 2010.

ESPACENET. Advanced Search. Disponível em: <http://ep.espacenet.com/ advancedSearch?locale=en_EP>. Acesso em: 08 jun. 2010.

FREEMAN, C. Network of innovators: a synthesis of research issues. Research Policy, v. 20, n. 5, p. 499-514, 1991.

GUARAU, C. Innovation networks in the biopharmaceutical sector: a study of UK small and medium sized enterprise. International Journal of Entrepreneurship and innovation management, v. 5, 2005.

IMAI, K.; BABA, Y. Systemic innovation and cross-border networks: transcending markets and hierarchies to create a new techno-economic system. OECD, Conference on Science Technology and Economic Growth. Paris, June 1989. 
JENSEN, J. R; HALVORSEN, K. E.; SHONNARD, D. R. Ethanol from lignocellulosics. U.S. federal energy and agricultural policy, and the diffusion of innovation. Biomass and Bioenergy, In Press, 2010.

LEITE, R. C. de C.; LEAL, M. R. L. V. O biocombustível no Brasil. Novos Estudos, São Paulo, Cebrap, n. 78, jul. 2007.

LEMOS, C. Inovação na era do conhecimento. In: LASTRES, H. M. M.; ALBAGLI, S. (Orgs.). Informação e globalização na era do conhecimento. Rio de Janeiro: Campus, 1999, cap. 5, p. 122-144.

MACEDO, I. C. Situação atual e perspectivas do etanol. Revista Estudos Avançados, v. 21, n. 59, 2007.

MACEDO, I. C.; SEABRA, J. E. A.; SILVA, J. E. A. R. Green house gases emissions in the production and use of ethanol from sugarcane in Brazil: the 2005/2006 averages and a prediction for 2020. Biomass and Bioenergy, London, UK, v. 32, n. 7, p. 582-595, 2008.

NEVES, M. F; TROMBIN, V. G.; CONSOLI, M. A. Mapeamento e quantificação do setor sucroenergético - versão preliminar. Ribeirão Preto: Markestrat/Fundace, 2009.

PELLEGRIN, I. de et al. Redes de inovação: construção e gestão da cooperação pró-inovação. R. Adm., São Paulo, v. 42, n. 3, p.313-325, jul./ago./set. 2007.

PORTO, G.S.; A decisão de cooperação universidade - empresa sob a ótica dos líderes de grupos de pesquisa da USP cadastrados no diretório de pesquisa do CNPQ. 2006. 187 p. Tese de Livre Docência - Faculdade de Economia, Administração e Contabilidade de Ribeirão Preto FEA-RP/USP , Departamento de Administração, Ribeirão Preto, 2006.

PYKA, A.; KUPPERS, G. (Eds.). Innovation networks - theory and practices. Edward Elgar Publishing, 2003.

RICHARDSON, R. J. Pesquisa social: métodos e técnicas. 3. ed. São Paulo: Atlas, 1999.

RYCROFT, R. W.; KASH, D. E. Self-organizing innovation networks: implications for globalization. Technovation, v. 24, p. 187-197, 2004.

SACHS, I. Da civilização do petróleo a uma nova civilização verde. Revista Estudos Avançados, v. 19, n. 55, 2005.

SCHUMPETER, J. A. Teoria do desenvolvimento econômico. 3 ed. São Paulo: Nova Cultural, 1984.

TRETYAK, O. A. Network: a new phenomenon of coordination and management. Russian European Center for Economic Policy. Jul. 2001.

U.S. DEPARTMENT OF ENERGY. Renewable Energy Annual 2008: Report. Washington, DC: U.S. Energy Information Administration (EIA), August 2010, 134 p. 


\section{Anexo}

Instituições participantes do Biomass Program

com projetos relacionados a etanol celulósico

\begin{tabular}{|c|c|}
\hline Instituição & $\begin{array}{c}\text { Nome de } \\
\text { identificação } \\
\text { na rede }\end{array}$ \\
\hline National Renewable Energy Laboratory & Inst1 \\
\hline Scripps Research Institute & Inst2 \\
\hline Weizmann Research Institute & Inst3 \\
\hline Pacific Northwest National Laboratory & Inst4 \\
\hline The Georgia Institute of Technology & Inst5 \\
\hline USDA Forest Products Laboratory & Inst6 \\
\hline U.S. Dept. of Agriculture & Inst7 \\
\hline U.S. Dept. of Energy & Inst8 \\
\hline State of Georgia & Inst9 \\
\hline Oak Ridge National Laboratory (ORNL) & Inst10 \\
\hline New York State Energy Research and Development Authority (NYSERDA) & Inst11 \\
\hline New York State Power Authority (NYPA) & Inst12 \\
\hline Midwest Laboratories & Inst13 \\
\hline Inc. Wallace Foundation & Inst14 \\
\hline National Corn Growers Association & Inst15 \\
\hline lowa Corn Promotion Board & Inst16 \\
\hline Fuels Technology Program & Inst17 \\
\hline Great Plains Institute for Sustainable Development & Inst18 \\
\hline Idaho National Laboratory & Inst19 \\
\hline Kentucky Office of Energy Policy & Inst20 \\
\hline New York State Energy Research and Development Authority (NYSERDA) & Inst21 \\
\hline Office of FreedomCAR and Vehicle Technologies & Inst22 \\
\hline Auburn University & Inst23 \\
\hline Dartmouth College & Inst24 \\
\hline Michigan State University & Inst25 \\
\hline
\end{tabular}

(continua) 
Instituições participantes do Biomass Program

com projetos relacionados a etanol celulósico

\begin{tabular}{|c|c|}
\hline Instituição & $\begin{array}{c}\text { Nome de } \\
\text { identificação } \\
\text { na rede }\end{array}$ \\
\hline Purdue University & Inst26 \\
\hline Texas A\&M University & Inst27 \\
\hline University of British Columbia & Inst28 \\
\hline University of California Riverside & Inst29 \\
\hline Cornell University & Inst30 \\
\hline University of Arkansas & Inst31 \\
\hline Colorado State University & Inst32 \\
\hline University of Colorado & Inst33 \\
\hline University of California & Inst34 \\
\hline lowa State University & Inst35 \\
\hline University of Louisville & Inst36 \\
\hline University of Kentucky Research foundation & Inst37 \\
\hline Mississippi State University & Inst38 \\
\hline University of North Dakota's Energy \& Environmental Research Center & Inst39 \\
\hline South Dakota State University & Inst40 \\
\hline Davis (UCD) & Inst41 \\
\hline Seattle University & Inst42 \\
\hline University of Sherbrooke, Canadá & Inst43 \\
\hline University of Florida & Inst44 \\
\hline Valero Energy Corporation & Inst45 \\
\hline ICM, Inc. & Inst46 \\
\hline INEOS New Planet Bio Energy & Inst47 \\
\hline Novozymes Biotech, Inc. & Inst48 \\
\hline Logos Technologies, Inc. & Inst49 \\
\hline Solazyme, Inc. & Inst50 \\
\hline
\end{tabular}


Instituições participantes do Biomass Program

com projetos relacionados a etanol celulósico

Instituição

Nome de identificação na rede

UOP LLC

Inst51

Renewable Energy Group, Inc.

Inst52

Abengoa Bioenergy Corp.

Inst53

BlueFire Ethanol, Inc.

Inst54

CH2M HILL

Inst55

GreenWood Resources

Inst56

Andritz

Inst57

Davy Process Technology

Inst58

Abener North America

Inst59

Poet Project Liberty

Inst60

IOGEN Corporation

Inst61

Cargill Dow LLC

Inst62

Diversa Corporation

Inst63

Deere \& Company

Inst64

Mat, Inc.

Inst65

Masada OxyNol, LLC

Inst66

Harris Group

Inst67

Lizan Process Solutions

Inst68

Tennessee Valley Authority

Inst69

Broin and Associates, Inc.

Inst70

Archer Daniels Midland Company

Inst71

BC International Corporation

Inst72

Dyadic International, Inc.

Inst73

Idaho National Laboratory

Inst74

Archer Daniels Midland (ADM)

Inst75

Elevance Renewable Sciences

Inst76 
Instituições participantes do Biomass Program

com projetos relacionados a etanol celulósico

\begin{tabular}{|c|c|}
\hline Instituição & $\begin{array}{c}\text { Nome de } \\
\text { identificação } \\
\text { na rede }\end{array}$ \\
\hline Enerkem Corporation & Inst77 \\
\hline Pacific Ethanol, Inc & Inst78 \\
\hline Lignol Innovations Inc & Inst79 \\
\hline Verenium Corporation & Inst80 \\
\hline Iroquois Bio-Energy Co., LLC & Inst81 \\
\hline AGCO & Inst82 \\
\hline AgraPure, Inc. & Inst83 \\
\hline Algenol Biofuels, Inc. & Inst84 \\
\hline American Process, Inc. & Inst85 \\
\hline Aventine Renewable Energy, Inc. & Inst86 \\
\hline Bioengineering Resources, Inc. & Inst87 \\
\hline Burns \& McDonnell Engineering Company, Inc. & Inst88 \\
\hline Case IH & Inst89 \\
\hline Chippewa Valley Ethanol Company & Inst90 \\
\hline E. I. Du Pont de Nemours \& Co., Inc. & Inst91 \\
\hline Genencor International & Inst92 \\
\hline Haldor Topsoe, Inc. & Inst93 \\
\hline Iron Horse Farms & Inst94 \\
\hline John Deere & Inst95 \\
\hline Katzen International, Inc. & Inst96 \\
\hline Mascoma Corporation & Inst97 \\
\hline Membrane Technology \& Research, Inc & Inst98 \\
\hline Michigan Economic Development Corporation (MEDC) & Inst99 \\
\hline Mississippi Ethanol, LLC & Inst100 \\
\hline New Planet Energy & Inst101 \\
\hline Range Fuels & Inst102 \\
\hline Sealaska Corporation & Inst103 \\
\hline
\end{tabular}


Instituições participantes do Biomass Program

com projetos relacionados a etanol celulósico

\begin{tabular}{lc}
\hline \multicolumn{1}{c|}{ Instituição } & $\begin{array}{c}\text { Nome de } \\
\text { identificação } \\
\text { na rede }\end{array}$ \\
\hline SunOpta, Inc. & Inst104 \\
The Dow Chemical Company & Inst105 \\
Vermeer & Inst106 \\
ZeaChem, Inc. & Inst107 \\
BlueFire Renewables & Inst108 \\
U.S. Department of Energy & Inst110 \\
Mississippi Alternative Energy Alliance & Inst111 \\
\hline
\end{tabular}

\section{Instituições participantes do Programa Bioen com projetos envolvidos com etanol celulósico}

\begin{tabular}{|c|c|}
\hline Instituição & $\begin{array}{l}\text { Nome de identificação } \\
\text { na rede }\end{array}$ \\
\hline Associação Brasileira de Tecnologia de Luz Síncrotron & ABTLS \\
\hline $\begin{array}{l}\text { Centro de Biologia Molecular e Engenharia Genética/Universidade } \\
\text { Estadual de Campinas (Unicamp) }\end{array}$ & CBMEG/Unicamp \\
\hline $\begin{array}{l}\text { Centro de Ciências Exatas e de Tecnologia/Universidade Federal de } \\
\text { São Carlos (UFSCar) }\end{array}$ & CCET/UFSCar \\
\hline $\begin{array}{l}\text { Centro de Ecofisiologia e Biofísica/Instituto Agronômico de } \\
\text { Campinas (IAC) }\end{array}$ & CEFBF/IAC \\
\hline $\begin{array}{l}\text { Centro de Energia Nuclear na Agricultura/Universidade de São } \\
\text { Paulo (Cena/USP) }\end{array}$ & Cena/USP \\
\hline $\begin{array}{l}\text { Centro de Engenharia, Modelagem e Ciências Sociais Aplicadas/ } \\
\text { Universidade Federal do ABC (UFABC) }\end{array}$ & CEMCSA/UFABC \\
\hline $\begin{array}{l}\text { Centro de Tecnologia das Radiações/Instituto de Pesquisas } \\
\text { Energéticas e Nucleares (Ipen) }\end{array}$ & CTR/Ipen \\
\hline Centro Nacional de Pesquisa em Energia e Materiais (CNPEM) & CNPEM \\
\hline Dedini & Dedini \\
\hline $\begin{array}{l}\text { Departamento de Física da Universidade Federal de Juiz de Fora } \\
\text { (UFJF) }\end{array}$ & DF/UFJF \\
\hline $\begin{array}{l}\text { Departamento de Microbiologia da Universidade Federal de Minas } \\
\text { Gerais (UFMG) }\end{array}$ & DMB/UFMG \\
\hline
\end{tabular}


Instituições participantes do Programa Bioen com projetos envolvidos com etanol celulósico

\begin{tabular}{|c|c|}
\hline Instituição & $\begin{array}{l}\text { Nome de identificação } \\
\text { na rede }\end{array}$ \\
\hline DEQ/UFRGS & DEQ/UFRGS \\
\hline Embrapa & Embrapa \\
\hline Engenharia Química UFSCar & EQ/UFSCar \\
\hline $\begin{array}{l}\text { Escola de Artes, Ciências e Humanidades/Universidade de São } \\
\text { Paulo (USP Leste) }\end{array}$ & EACH/USP Leste \\
\hline Escola de Engenharia de Lorena/Universidade de São Paulo (USP) & EEL/USP \\
\hline Escola Politécnica/Universidade de São Paulo (USP) & EP/USP \\
\hline $\begin{array}{l}\text { Faculdade de Ciências Farmacêuticas de Ribeirão Preto/ } \\
\text { Universidade de São Paulo (USP) }\end{array}$ & FCFRP/USP \\
\hline $\begin{array}{l}\text { Faculdade de Engenharia de Alimentos Universidade Estadual de } \\
\text { Campinas (FEA/Unicamp) }\end{array}$ & FEA/Unicamp \\
\hline $\begin{array}{l}\text { Faculdade de Engenharia Química/Universidade Estadual de } \\
\text { Campinas (FEQ/Unicamp) }\end{array}$ & FEQ/Unicamp \\
\hline Fapesp & Fapesp \\
\hline $\begin{array}{l}\text { Grupo de Ciências Espaciais e Atmosféricas/Instituto Nacional de } \\
\text { Pesquisas Espaciais (Inpe) }\end{array}$ & GCEA/Inpe \\
\hline Instituto de Biociências/Universidade de São Paulo (USP) & IBC/USP \\
\hline Instituto de Biologia/Universidade Estadual de Campinas (Unicamp) & IB/Unicamp \\
\hline $\begin{array}{l}\text { Instituto de Economia/Universidade Estadual de Campinas } \\
\text { (Unicamp) }\end{array}$ & IE/Unicamp \\
\hline $\begin{array}{l}\text { Instituto de Física de São Carlos/Universidade de São Paulo (IFSC/ } \\
\text { USP) }\end{array}$ & IFSC/USP \\
\hline Instituto de Pesquisas Tecnológicas (IPT) & IPT \\
\hline Instituto de Química de São Carlos/Universidade de São Paulo (USP) & IQSC/USP \\
\hline Instituto de Química/Universidade de São Paulo (USP) & IQ/USP \\
\hline Instituto de Química/Universidade Estadual Paulista (Unesp) & IQ/Unesp \\
\hline Instituto de Tecnologia de Alimentos (Ital) & Ital \\
\hline $\begin{array}{l}\text { Laboratório de Microbiologia/Universidade Estadual Paulista (Unesp } \\
\text { Rio Claro) }\end{array}$ & LMB/Unesp Rio Claro \\
\hline Laboratório Nacional de Ciência e Tecnologia do Bioetanol (CTBE) & СTBE \\
\hline Oxiteno & Oxiteno \\
\hline
\end{tabular}




\section{Instituições participantes do Programa Bioen com projetos envolvidos com etanol celulósico}

\begin{tabular}{l|l}
\hline \multicolumn{1}{c|}{ Instituição } & \multicolumn{1}{c}{$\begin{array}{c}\text { Nome de identificação } \\
\text { na rede }\end{array}$} \\
\hline PEQ/Coppe/UFRJ & PEQ/Coppe/UFRJ \\
Southern Cross University, Austrália & SCU Austrália \\
Universidade de São Paulo & USP \\
Universidade Federal de Santa Catarina & UFSC \\
Universidade Federal do ABC & UFABC \\
\hline
\end{tabular}

Geciane Silveira Porto - geciane@usp.br FEA-RP/USP

Avenida Bandeirantes, 3.900, Monte Alegre Ribeirão Preto, SP - 14040-905

Simone Vasconcelos Ribeiro Galina - svgalina@usp.br FEA-RP/USP

Avenida Bandeirantes, 3.900, Monte Alegre

Ribeirão Preto, SP - 14040-905 